\title{
Symmetric Fusion Wavelet Frames in Two Dimensions
}

\author{
Zhiqiang Li \\ Institute of Management Science and Engineering, Henan University, Kaifeng 475001, \\ Henan, P. R. China; \\ zhiqiangli0111@163.com
}

Keywords: Wavelet, The fusion wavelet frame; Symmetric property.

\begin{abstract}
Frame theory plays an important role in field of the engineering and technology because of their redundancy properties. In this paper, symmetric fusion wavelet frames with several generators are constructed from any fusion wavelet frames given, which generalizes the existing result to the case of several generators in higher dimension.
\end{abstract}

\section{Introduction}

The notion of frames was first found by Duffin and Schaeffer [1] when they researched about general Fourier series. Since paper of Daubechies, Grossmann, and Meyer [2] was published, the frame theory began to be widely used by all over the world. At first, frames have been used in signal processing and image processing [3]. Then, frames are used to shorten the effect of losses in communication systems and to improve the robustness of data transmission [4]. Also they could be used to design high-rate constellation in multiple-antenna code[5].

Wavelet frame is an important case of frame. Wavelet system is obtained by shifting and dilating some functions. In the 1980s, wavelets were introduced and developed by people. From then on, wavelets had obtained huge success in many promising asides.

Gabor frame is another important example about frame. Gabor systems were first by coined by Gabor in 1946[6]. They are generated by modulations and translations of some fixed functions.

In paper [7], authors introduced wave packet systems by fusing three operators of dilations, modulations and translations to the Gaussian function when they studying some singular integral operators. In paper [8], authors adopted the same expression to classfy any collections of functions which are obtained by applying the same operations. In this paper, we name wave packet systems as fusion wavelet systems for the sake of the simplicity. That is, let fixed functions $\left(g^{1}, g^{2}, \cdots, g^{M}\right) \subset L^{2}\left(R^{2}\right)$, A be invertible matix and define the fusion wavelet system as the following

$$
G(g, j, l, k, m)=\left\{D_{A}^{j} E_{l} T_{k} g^{m}: j \in Z, l, k \in Z^{2}, m=1,2, \cdots M\right\},
$$

where $D_{A} f(x)=\sqrt{\operatorname{det} A} f(A x), T_{k} f(x)=f(x-k)$ and $E_{l} f(x)=e^{2 \pi l x} f(x)$. In fact, Gabor systems and wavelet systems are special cases of fusion wavelet systems. Fusion wavelet systems have been used to some problems in harmonic analysis and operator theory [9, 10].

In paper [11], authors classified fusion wavelet systems by the theory of generalized shift invariant systems and gave a sufficient condition of fusion wavelet system to be a frame. In paper [12], authors presented more examples with better properties of fusion wavelet frames.

It is well known that the symmetry of wavelet plays an important role in applications. In [14], authors gave a good way to construct symmetric wavelet frames from any wavelet frames given. Motivated the paper [13], we will discuss the case of fusion wavelet systems in this paper. Of course, our way combines with some ways in wavelet analysis and Gabor analysis.

In this paper, symmetric fusion wavelet frames with several generators are constructed in higher dimension from any fusion wavelet frames given, which generalizes the existing result in [14] to the case of several generators in higher dimension. This way improves largely the amount of wavelet family. 


\section{Preliminaries}

Throughout this paper, the following notations will be used. $R$ and $Z$ denote the set of real numbers and the set of integers, respectively. $L^{2}\left(R^{2}\right)$ is the space of all square-integrable functions, and $\left\langle\cdot, \cdot>\right.$ and $\|\cdot\|$ denote the inner product and norm in $L^{2}\left(R^{2}\right)$, respectively, and $l^{2}(Z)$ denotes the space of all square summable sequences.

Let us recall the definition of frame.

Definition 2.1 Let $H$ be a separable Hilbert space. A sequence $\left\{f_{i}\right\}_{i \in N}$ of elements of $H$ is a frame for $H$ if there exist constants $0<C \leq D<\infty$ such that for all $f \in H$,we have

$$
C\|f\|^{2} \leq \sum_{i \in N}\left\|\left\langle f, f_{i}\right\rangle\right\|^{2} \leq D\|f\|^{2} .
$$

The numbers $C, D$ are called lower and upper frame bounds, respectively (the largest $C$ and the smallest $D$ for which (2.1) holds are the optimal frame bounds). Those sequences which satisfy only the upper inequality in (2.1) are called Bessel sequences.

Let $T_{f}$ denote the synthesis operator of $f=\left\{f_{i}\right\}_{i \in N}$, i.e., $T_{f}(c)=\sum_{i} c_{i} f_{i}$ for each sequence of scalars $c=\left(c_{i}\right)_{i \in N}$. Then the frame operator $S h=T_{f} T_{f}^{*}(h)$ associated with $\left\{f_{i}\right\}_{i \in N}$ is a bounded, invertible, and positive operator mapping of $H$ on itself. This provides the reconstruction formula

$$
h=\sum_{i=1}^{\infty}<h, g_{i}>f_{i}=\sum_{i=1}^{\infty}<h, f_{i}>g_{i}, \forall h \in H .
$$

where $g_{i}=S^{-1} f_{i}$. The family $\left\{g_{i}\right\}_{i \in N}$ is also a frame for $H$ and is called the canonical dual frame of $\left\{f_{i}\right\}_{i \in N}$. If $\left\{g_{i}\right\}_{i \in N}$ is any sequence in $H$ which satisfies

$$
h=\sum_{i=1}^{\infty}<h, g_{i}>f_{i}=\sum_{i=1}^{\infty}<h, f_{i}>g_{i}, \forall h \in H,
$$

it is called an alternate dual frame of $\left\{f_{i}\right\}_{i \in N}$.

Then, we will give the definitions of a fusion wavelet frame and the frame wavelet fusion functions..

Definition 2.2 We say that the fusion wavelet system defined by (1.1) is a fusion wavelet frame if it is a frame for $L^{2}\left(R^{2}\right)$. Then, the vector functions $\left(g^{1}, g^{2}, \cdots, g^{M}\right)$ is called the frame fusion wavelet functions.

\section{Symmetric Fusion Wavelet Frames}

In this section, we will construct symmetric fusion wavelet frames with several generators in higher dimension from any existing fusion wavelet frames.

For fixed function family $\left(g^{1}, g^{2}, \cdots, g^{M}\right) \subset L^{2}\left(R^{2}\right)$, define new symmetric or antisymmetric functions about origin as the following:

$$
g_{1}^{m}(x)=\frac{g^{m}(x)+g^{m}(-x)}{2}, g_{2}^{l}(x)=\frac{g^{m}(x)-g^{m}(-x)}{2} \text {. }
$$

Thus, we have

Theorem 3.1 Suppose that fusion wavelet system

$G(g, j, l, k, m)=\left\{D_{A}{ }^{j} E_{l} T_{k} g^{m}: j \in Z, l, k \in Z^{2}, m=1,2, \cdots M\right\}$,

defined by (1.1) is a frame for $L^{2}\left(R^{2}\right)$ with frame bounds $C_{1}, C_{2}$, then fusion wavelet system

$$
\left\{D_{A}{ }^{j} E_{l} T_{k} g_{1}{ }^{m} \cup D_{A}{ }^{j} E_{l} T_{k} g_{2}{ }^{m}: j \in Z, k, l \in Z^{2}, m=1,2, \cdots M\right\}
$$


is a symmetric frame for $L^{2}\left(R^{2}\right)$ about origin with frame bounds $C_{1}, C_{2}$, where the functions $g_{1}{ }^{m}(x), g_{2}{ }^{m}(x)$ are defined by (3.1).

Proof. Because fusion wavelet system

$G(g, j, l, k, m)=\left\{D_{A}{ }^{j} E_{l} T_{k} g^{m}: j \in Z, l, k \in Z^{2}, m=1,2, \cdots M\right\}$,

is a frame with frame bounds $C_{1}, C_{2}$, then, we have

$C_{1}\|f\|^{2} \leq\left.\sum_{m=1}^{M} \sum_{j \in Z} \sum_{l \in Z^{2}} \sum_{k \in Z^{2}}\left\langle f, D_{A}{ }^{j} E_{l} T_{k} g^{m}\right\rangle\right|^{2} \leq C_{2}\|f\|^{2}$.

In the following we will calculate the series

$\left.\sum_{m=1}^{M} \sum_{j \in Z} \sum_{l \in Z^{2}} \sum_{k \in Z^{2}}\left\langle f, D_{A}{ }^{j} E_{l} T_{k} g_{1}{ }^{m}\right\rangle\right|^{2}+\left.\sum_{m=1}^{M} \sum_{j \in Z} \sum_{l \in Z^{2}} \sum_{k \in Z^{2}}\left\langle f, D_{A}{ }^{j} E_{l} T_{k} g_{2}{ }^{m}\right\rangle\right|^{2}$.

From property of inner product, we can deduce

$\left|\left\langle f(\cdot), D_{A}{ }^{j} E_{l} T_{k} g_{1}{ }^{m}\right\rangle\right|^{2}=\left|\left\langle f(\cdot), D_{A}{ }^{j} E_{l} T_{k} \frac{g^{m}(\cdot)}{2}\right\rangle+\left\langle f(\cdot), D_{A}{ }^{j} E_{l} T_{k} \frac{g^{m}(-\cdot)}{2}\right\rangle\right|^{2}$.

For any complex numbers $z_{1}, z_{2}$, it is well known that the following equality holds $\left|z_{1}+z_{2}\right|^{2}=\left|z_{1}\right|^{2}+\left|z_{2}\right|^{2}+z_{1} \overline{z_{2}}+\overline{z_{1}} z_{2}$.

From (3.5) and (3.6), we have

$$
\begin{aligned}
\left.\left\langle f(\cdot), D_{A}{ }^{j} E_{l} T_{k} g_{1}{ }^{m}(\cdot)\right\rangle\right|^{2} & =\frac{1}{4}\left|\left\langle f(\cdot), D_{A}{ }^{j} E_{l} T_{k} g^{m}(\cdot)\right\rangle\right|^{2}+\frac{1}{4}\left|\left\langle f(\cdot), D_{A}{ }^{j} E_{l} T_{k} g^{m}(-\cdot)\right\rangle\right|^{2} \\
& +\frac{1}{4}\left\langle f(\cdot), D_{A}{ }^{j} E_{l} T_{k} g^{m}(\cdot)\right\rangle \overline{\left\langle f(\cdot), D_{A}{ }^{j} E_{l} T_{k} g^{m}(-\cdot)\right\rangle} \\
& +\frac{1}{4} \overline{\left\langle f(\cdot), D_{A}{ }^{j} E_{l} T_{k} g^{m}(\cdot)\right\rangle}\left\langle f(\cdot), D_{A}{ }^{j} E_{l} T_{k} g^{m}(-\cdot)\right\rangle .
\end{aligned}
$$

In the similar way, we can prove

$$
\begin{aligned}
\left.\left\langle f(\cdot), D_{A}{ }^{j} E_{l} T_{k} g_{2}{ }^{m}(\cdot)\right\rangle\right|^{2} & =\frac{1}{4}\left|\left\langle f(\cdot), D_{A}{ }^{j} E_{l} T_{k} g^{m}(\cdot)\right\rangle\right|^{2}+\frac{1}{4}\left|\left\langle f(\cdot), D_{A}{ }^{j} E_{l} T_{k} g^{m}(-\cdot)\right\rangle\right|^{2} \\
& -\frac{1}{4}\left\langle f(\cdot), D_{A}{ }^{j} E_{l} T_{k} g^{m}(\cdot)\right\rangle\left\langle\overline{\left\langle f(\cdot), D_{A}{ }^{j} E_{l} T_{k} g^{m}(-\cdot)\right\rangle}\right. \\
& -\frac{1}{4} \overline{\left\langle f(\cdot), D_{A}{ }^{j} E_{l} T_{k} g^{m}(\cdot)\right\rangle}\left\langle f(\cdot), D_{A}{ }^{j} E_{l} T_{k} g^{m}(-\cdot)\right\rangle .
\end{aligned}
$$

Comparing with (3.7) and (3.8), we get

$$
\begin{aligned}
\sum_{m=1}^{M} \sum_{j \in Z} & \left.\sum_{l \in Z^{2}} \sum_{k \in Z^{2}}\left\langle f, D_{A}{ }^{j} E_{l} T_{k} g_{1}{ }^{m}\right\rangle\right|^{2}+\left.\sum_{m=1}^{M} \sum_{j \in Z} \sum_{l \in Z^{2}} \sum_{k \in Z^{2}}\left\langle f, D_{A}{ }^{j} E_{l} T_{k} g_{2}{ }^{m}\right\rangle\right|^{2} \\
= & \frac{1}{2} \sum_{m=1}^{M} \sum_{j \in Z} \sum_{l \in Z^{2}} \sum_{k \in Z^{2}}\left|\left\langle f(\cdot), D_{A}{ }^{j} E_{l} T_{k} g^{m}(\cdot)\right\rangle\right|^{2} \\
& +\frac{1}{2} \sum_{m=1}^{M} \sum_{j \in Z} \sum_{l \in Z^{2}} \sum_{k \in Z^{2}}\left|\left\langle f(\cdot), D_{A}{ }^{j} E_{l} T_{k} g^{m}(-\cdot)\right\rangle\right|^{2}
\end{aligned}
$$

By simple calculation, we get

$\sum_{m=1}^{M} \sum_{j \in Z} \sum_{l \in Z^{2}} \sum_{k \in Z^{2}}\left|\left\langle f(\cdot), D_{A}{ }^{j} E_{l} T_{k} g^{m}(-\cdot)\right\rangle\right|^{2}=\sum_{m=1}^{M} \sum_{j \in Z} \sum_{l \in Z^{2}} \sum_{k \in Z^{2}}\left|\left\langle f(-\cdot), D_{A}{ }^{j} E_{l} T_{k} g^{m}(\cdot)\right\rangle\right|^{2}$.

According to (3.3), we obtain

$C_{1}\|f(-\cdot)\|^{2} \leq\left.\sum_{m=1}^{M} \sum_{j \in Z} \sum_{l \in Z^{2}} \sum_{k \in Z^{2}}\left\langle f(-\cdot), D_{A}{ }^{j} E_{l} T_{k} g^{m}\right\rangle\right|^{2} \leq C_{2}\|f(-\cdot)\|^{2}$,

From (3.10), (3.11) and the equality $\|f(-\cdot)\|^{2}=\|f(\cdot)\|^{2}$, we deduce 


$$
C_{1}\|f\|^{2} \leq\left.\sum_{m=1}^{M} \sum_{j \in Z} \sum_{l \in Z^{2}} \sum_{k \in Z^{2}}\left\langle f, D_{A}{ }^{j} E_{l} T_{k} g^{m}(-\cdot)\right\rangle\right|^{2} \leq C_{2}\|f\|^{2} .
$$

At last, comparing with (3.3), (3.9) and (3.12), we obtain

$$
C_{1}\|f\|^{2} \leq\left.\sum_{m=1}^{M} \sum_{j \in Z} \sum_{l \in Z^{2}} \sum_{k \in Z^{2}}\left\langle f, D_{A}{ }^{j} E_{l} T_{k} g_{1}{ }^{m}\right\rangle\right|^{2}+\left.\sum_{m=1}^{M} \sum_{j \in Z} \sum_{l \in Z^{2}} \sum_{k \in Z^{2}}\left\langle f, D_{A}{ }^{j} E_{l} T_{k} g_{2}{ }^{m}\right\rangle\right|^{2} \leq C_{2}\|f\|^{2}
$$

As a conclusion, we have obtained Theorem 3.1.

\section{Summary}

Wavelet analysis has obtained huge achievement in signal processing, denoising, sampling theorem, applied mathematics and other fields. In this paper, symmetric fusion wavelet frames with several generators in higher dimension are constructed from any existing fusion wavelet frames, which generalizes the existing result to the case of several generators and higher dimensions. This way improves the amount of wavelets largely.

\section{References}

[1] R. Duffin, A. Schaeffer, A class of nonharmonic Fourier series. Trans. Amer. Math. Soc. 72 (1952) 341-366.

[2] I. Daubechies, A. Groddmann, Y. Mayer, Painless nonorthogonal expansions, J. Math. Phys. 27 (1986) 1271-1283.

[3] P. Casazza, J. Kovacevic, J. Kelner, Equal-norm tight frames with erasures, Adv. Comput. Math.18 (2003) 387-430.

[4] V. Goyal, J. Kovacevic, J. Kelner, Quantized frames expansions with erasures. Appl. Comput. Harmon. Anal. 10 (2001) 203-233.

[5] B. Hassibi, B. Hochwald, A. Shokrollahi, W. Sweldens, Representation theory for high-rate multiple-antenna code design, IEEE Trans. Inform. Theory 47 (2001) 2335-2367.

[6] D. Gabor, Thoery of communications. J. Inst. Elec. Engrg. 93 (1946) 429-457.

[7] A. Cordoba and C. Fefferman. Wave packets and Fourier integral operators. Comm. Partial Differrential Equations 3 (1978) 979-1005.

[8] D. Labate, G. Weiss and E. Wilson. An approach to the study of wave packet systems. Contemp. Math., Wavelets, Frames and Operator Theory 345 (2004) 215-235.

[9] M. Lacey and C. Thiele, $L^{p}$ estimates on the bilinear Hilbert transform for $2<p<\infty$. Ann. of Math. 146 (1997) 693-724.

[10] M. Lacey and C. Thiele. On Caldern’s conjecture. Ann. of Math. 149 (1999) 475-496.

[11] O. Christensen and A. Rahimi. Frame properties of wave packet systems in $L^{2}\left(R^{d}\right)$. Adv. Comput. Math. 29 (2008) 101-111.

[12] E. Hernandez, D. Labate, G. Weiss and E. Wilson. Oversampling, quasi affine frames and wave packets. Appl. Comput. Harmon. Anal. 16 (2004) 111-147.

[13] S. Goh, Z. Lim, Z. Shen, Symmetric and antisymmetric tight wavelet frames, Appl. Comput. Harmon. Anal. 20 (2006) 411-421.

[14] G.Wu and H. Cao, Symmetric wave packet frames, Applied Mechanics and Materials 227-429 (2013) 1528-1531. 